\title{
Evaluar para aprender: hacer más compleja la tarea a los alumnos
}

\author{
Evaluate to learn: Make the task more complex for students
}

\author{
Yolanda Chávez Ruiz ${ }^{1}$ \\ Felipe Martínez Rizo
}

\begin{abstract}
Resumen: El propósito de este estudio está centrado en caracterizar las prácticas de enseñanza-evaluación (como dos elementos indisociables), a partir de la exigencia cognitiva de las tareas que proponen los profesores a sus alumnos para enseñar un contenido matemático. En este estudio de corte cualitativo observamos y videograbamos 12 clases de matemáticas de dos maestros de escuelas primarias en Aguascalientes, México; el registro se hizo en un instrumento guía, diseñado para este estudio. La observación de las prácticas mostró que, aunque las tareas propuestas a los estudiantes con base en libros de texto y otros materiales tengan un alto potencial matemático, es difícil para los docentes mantener altos niveles de exigencia cognitiva durante el desarrollo de las clases.
\end{abstract}

Palabras clave: enseñanza de las matemáticas; evaluación formativa; exigencia cognitiva; tareas matemáticas.

Abstract: The purpose of this study is to characterize teaching-assessment practices (as two inseparable elements), based on the cognitive requirement of the tasks proposed by teachers to their students regarding a mathematical

Fecha de recepción: 24 de junio de 2017. Fecha de aceptación: 3 de septiembre de 2018.

1 Instituto de Educación de Aguascalientes, México. yolachavezruiz@gmail.com orcid.org/0000-00030955-4803.

2 Departamento de Educación, Universidad Autónoma de Aguascalientes, México. felipemartinez.rizo@ gmail.com orcid.org/0000-0002-7519-4247. 
content. In this qualitative study, 12 math lessons by two elementary school teachers were observed and videotaped in Aguascalientes, Mexico; the record was made in a guiding instrument designed for this study. The observation of the practices showed that, although the tasks proposed to students based on textbooks and other materials have a high mathematical potential, it is difficult for teachers to maintain high levels of cognitive demand during the development of the classes.

Keywords: teaching of mathematics; formative evaluation; cognitive requirement; mathematical tasks.

\section{INTRODUCCIÓN}

Evaluar es formular juicios sobre el valor de algo, aunque no se hagan públicos y solo sirvan para normar la propia acción. De manera consciente o no, el profesor hace continuamente juicios sobre lo que aprenden los alumnos y estos juicios lo llevan a tomar ciertas decisiones durante las clases.

Los profesores están familiarizados con varios tipos de evaluación: a) Según el momento en que la efectúan: inicial, intermedia y final; b) Según los agentes que intervienen: autoevaluación, coevaluación y heteroevaluación; c) Según el propósito que se persigue: sumativa y formativa (Casanova, 1998; García, Aguilera, Pérez y Muñoz, 2011; Martínez-Rizo, 2012; Ravela, Picaroni y Loureiro, 2017). Respecto del último punto, conviene señalar que la evaluación sumativa es aquella que se limita a la formulación del juicio sobre los niveles de aprendizaje alcanzados por los estudiantes, mientras que la evaluación formativa (en adelante EF) busca además contribuir a que esos niveles mejoren. Los maestros utilizan diversos tipos de evaluación; sin embargo, en las interacciones que ocurren en el aula entre profesor, alumnos y contenidos es posible que el docente en realidad no identifique la evaluación como una herramienta que promueve el aprendizaje de los niños (Martínez-Rizo, 2012; Ravela et al., 2017).

En las últimas décadas ha habido un incremento notable de investigaciones sobre enseñanza y aprendizaje de las matemáticas (Niss, 1993; Sánchez, 2002; Llinares, S. y Krainer, K., 2006; Ávila, A., 2004), pero el estudio sobre las prácticas de evaluación en la enseñanza de esta materia no ha tenido tanto desarrollo (Niss, 1993; Clark, 2006). 
Es frecuente que la evaluación se entienda como una actividad diferente de la enseñanza, que tiene lugar después de esta, pero una concepción más rica (García et al., 2011; Martínez-Rizo, 2012), en particular de la EF, reconoce que enseñanza y evaluación son dos aspectos de una misma actividad: el maestro enseña y al mismo tiempo evalúa, al observar lo que hacen sus alumnos, sus procesos de resolución, sus maneras de involucrarse con la tarea matemática, permitiendo ajustar la enseñanza al detectar si los estudiantes están alcanzando o no los aprendizajes esperados.

Por ello, en este trabajo retomamos la EF como elemento indisociable de la práctica de enseñanza, que potencialmente contribuye al aprendizaje de los alumnos y no se limita a utilizar los resultados para efectos de calificación o selección (Cfr. Martínez Rizo, 2012). Entendemos la EF como el medio que permite a los profesores obtener información sobre la forma en que los estudiantes comprenden distintos contenidos matemáticos y encaran el potencial matemático de las tareas, así como identificar las posibles dificultades y aciertos de los alumnos en los procesos de aprendizaje, para estar en posibilidad de darles un seguimiento y apoyo.

Por otra parte, tanto la enseñanza como la evaluación pueden referirse a aprendizajes con diferente nivel de complejidad. En el caso de las matemáticas, en los libros de texto gratuito ${ }^{3}$ que distribuye la Secretaría de Educación Pública (SEP), las tareas propuestas para que trabajen los estudiantes tienen, en muchos casos, un alto potencial matemático, pero la forma concreta en que los profesores gestionan la clase puede hacer que el trabajo de los alumnos se reduzca a actividades de baja exigencia cognitiva; investigaciones como la coordinada por Ávila (2004) muestran cómo los maestros reducen los alcances cognitivos de las tareas señaladas en los libros de texto gratuito que la SEP distribuye.

El estudio reporta el resultado de la observación de clases de matemáticas de dos maestros de educación primaria de cuarto y quinto grado, para identificar el nivel cognitivo de las tareas propuestas y la manera en que el potencial de la actividad matemática que realizan los alumnos, al enfrentar dichas tareas es modificado por la forma en que los docentes reaccionan a partir de lo que detectan en la actividad de los estudiantes, o sea debido a la retroalimentación

\footnotetext{
${ }^{3}$ Estos libros fueron los llamados Desafíos matemáticos, que a partir del 2012 los profesores y alumnos de primaria en México tuvieron a su disposición, primero como un material de apoyo y complemento del libro de texto de matemáticas y posteriormente como único texto para la asignatura de Matemáticas en los seis grados de educación primaria. Además del libro para el alumno, los profesores cuentan con un libro para el maestro, que incluye recomendaciones didácticas.
} 
en que se concreta la EF; en otras palabras, observamos las interacciones de enseñanza y evaluación que ocurren en la clase de matemáticas.

\section{REFERENTES TEÓRICOS}

En este apartado presentamos conceptos de dos cuerpos de literatura: la EF y la didáctica de las matemáticas; así pueden apreciarse los puntos de contacto de ambas tradiciones como dos caras de una misma actividad de los docentes, que son la enseñanza y la evaluación.

Un importante punto común es el de la demanda cognitiva de las actividades de enseñanza y de evaluación, que ahora tienden a tener un nivel más alto que en el pasado. El papel actual del profesor de primaria no es el mismo que el que se le asignaba hasta los años 60 del siglo pasado, cuando solamente se esperaba que mantuviera en orden a sus alumnos y enseñara cuestiones básicas de aritmética, a leer y escribir, además de memorizar algunos temas.

Hoy las cosas han cambiado: el maestro debe preparar a los estudiantes para enfrentar conocimientos que aún no existen o están en desarrollo, y ayudarlos a adquirir las competencias y habilidades necesarias para enfrentarse a formas emergentes de organización y comunicación. Al profesor se le piden muchas cosas: planear; recabar información e interpretarla; diseñar o elegir situaciones y ejercicios para trabajar con sus alumnos; anticipar procedimientos de solución, errores y dificultades que podrían tener los estudiantes; también se le exige promover el aprendizaje mediante la asignación de tareas, observar los procesos de aprendizaje y tomar decisiones con base en ello (Cfr. SEP, 2011), por lo que la EF puede ser una importante herramienta en este proceso.

A diferencia de la sumativa, la EF no se limita a dar una calificación: busca contribuir a que los alumnos mejoren; por ello lo que la distingue es la calidad de la retroalimentación que ofrece, y éste es un aspecto fundamental en los procesos de enseñanza y de aprendizaje. Cabe aclarar que la retroalimentación no es el único aspecto de la $\mathrm{EF}$, aunque en ocasiones sean consideras como sinónimos; más adelante lo explicamos detalladamente.

De acuerdo con Martínez-Rizo (2012), la EF es aquella que contribuye a que los estudiantes aprendan, por lo cual una buena retroalimentación no se limita a informar si el alumno domina o no un tema, sino que contribuye a que avancen en el proceso de aprendizaje y ayuda a transitar del punto en que se encuentran, al definido como meta del proceso (Martínez-Rizo, 2012: 23). En este 
sentido, la EF es indisociable de la enseñanza: “... la evaluación formativa, o para el aprendizaje, sucede mientras el aprendizaje va en marcha con la intención de planear el siguiente paso en la enseñanza, en especial, para proporcionarle al alumno retroalimentación a fin de mejorar la calidad de su trabajo y ayudarlo a sentir el control de su proceso de aprendizaje" (García et al., 2011: 18).

Es importante advertir que el tipo de retroalimentación que un docente puede brindar a los alumnos depende del nivel de demanda cognitiva de los aprendizajes esperados. Si se trata de memorizar unos datos y un alumno solo consigue recordar una parte, no se le puede decir mucho más, solo que le falta otra parte y que lo siga intentando. En cambio, si se trata de resolver un problema de cierta complejidad, el profesor puede usar diferentes combinaciones de estrategias para que los niños utilicen diversos recursos y procesos de solución, es decir, hay amplio espacio para una retroalimentación rica.

Si el profesor propone una tarea matemática a los estudiantes y ellos se involucran en el trabajo, la retroalimentación que el maestro ofrece puede ser diferente. Frases como "Fíjate bien, Continúa mejorando, ¿Estás seguro de que es correcto?, Así no es, Échale ganas, Tú puedes hacerlo mejor, Lee bien la consigna", dan retroalimentación pero no de tipo formativo, ya que no ofrecen al estudiante información específica sobre su desempeño en cuanto a la calidad de su trabajo y cómo mejorarlo.

\section{TAREAS MATEMÁTICAS Y EXIGENCIA COGNITIVA}

Uno de los recursos que el docente utiliza para el aprendizaje de matemáticas son las tareas, por lo cual para entender lo que sucede en las aulas es importante caracterizarlas, así como las intervenciones del maestro durante el tiempo en que los alumnos llevan a cabo dichas tareas.

Entendemos por tarea matemática el conjunto de actividades organizadas y orientadas, con una o múltiples estrategias de solución, donde es posible utilizar diversas representaciones, lo cual permite a los estudiantes involucrarse con la actividad matemática. De acuerdo con Penalva y Llinares (2011) las tareas matemáticas son muy importantes, pues determinan lo que los estudiantes pueden llegar a aprender.

En una clase de matemáticas, los profesores de primaria suelen plantear una o varias tareas, y las pueden formular de manera oral o escrita, partiendo de un verbo en imperativo: Calcula..., Resuelve..., Construye... Las tareas también pueden 
formularse a partir de preguntas: ¿Cuántos...? ¿Cuál es la relación entre...? (Niss, 1993). Pueden, entonces, plantearse tareas mediante diferentes recursos: preguntas, ejercicios, problemas, proyectos.

Investigaciones realizadas durante los últimos 25 años han mostrado que los alumnos aprenden mejor cuando están en aulas donde se mantiene un alto nivel de exigencia cognitiva en las tareas que resuelven.

El potencial de la tarea matemática está relacionado con el nivel de exigencia cognitiva, que es "el tipo y nivel de pensamiento requerido de los estudiantes para poder participar en la tarea y resolverla con éxito" (Stein, Smith, Henningsen, Silver, en Benedicto y Gutiérrez, 2015: 154). En este sentido, el tipo y nivel de pensamiento con el cual se involucran los niños determina su aprendizaje, por lo que

las tareas tienen que corresponder con los objetivos de aprendizaje; por ejemplo si lo que el maestro pretende es aumentar la habilidad y la eficacia para que los alumnos recuerden reglas y definiciones, entonces puede proponer tareas centradas en la memorización; si su objetivo es aumentar la rapidez y la exactitud de los alumnos al resolver operaciones, puede proponer tareas de ejercitación como el cálculo mental, si su propósito es hacer que los estudiantes se impliquen en formas de razonamiento complejas y desarrollen destrezas de comunicación, es necesario proponer tareas de alto nivel de exigencia cognitiva (Penalva y Llinares, 2011: 31).

Para clasificar las tareas y las intervenciones que los profesores plantean a sus alumnos, en este trabajo utilizamos, con ajustes, la taxonomía de Smith y Stein (1998), que proponen clasificarlas en cuatro niveles de exigencia cognitiva: tareas de memorización, de procedimientos sin conexión, de procedimientos con conexión, o para hacer matemáticas. El NCTM (National Council of Teachers of Mathematics, 2014) retoma estas categorías para clasificar las tareas matemáticas, ubicando las dos primeras como de bajo nivel de complejidad, mientras que las dos últimas son de alto nivel de complejidad. Enseguida se describen esos cuatro niveles, con ejemplos tomados de observaciones reales en diversas clases de matemáticas con profesores de educación primaria en México. ${ }^{4}$

\footnotetext{
4 Algunos ejemplos son de esta investigación y otros del repertorio de clases observadas por los investigadores participantes.
} 


\section{Tareas de memorización}

Son actividades en las cuales el alumno recupera la información de que dispone; en las tareas de este tipo se solicita a los estudiantes que hagan uso de su memoria para reproducir hechos, reglas, fórmulas o definiciones, generalmente respondiendo a preguntas cerradas; también pueden utilizarse representaciones gráficas o simbólicas sencillas. No son ambiguas, implican la reproducción exacta de lo visto previamente.

Un ejemplo de tareas de este tipo es el siguiente:

"Colorea $1 / 4$ de la figura"

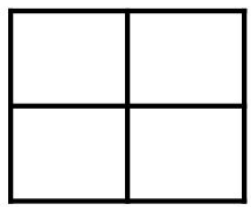

\section{Tareas de procedimiento sin conexión}

En estas tareas el alumno muestra comprensión del contenido matemático que le permite resolver tareas simples. Son tareas que usan algoritmos que se expresan de manera específica o una instrucción previa de otra tarea, indican qué hay que hacer y cómo hacerlo; implican una exigencia cognitiva limitada para la conclusión con éxito de la tarea; existe poca ambigüedad; no hay conexiones con los conceptos o significados en que se basa el procedimiento; las respuestas de los alumnos deben ser correctas; en lugar de explicar la comprensión del proceso matemático, solo se describe el procedimiento. Una tarea de este tipo puede ser como la siguiente:

"Divide en cuatro partes la siguiente figura y colorea $3 / 4$ " 


\section{Tareas de procedimiento con conexión}

Cuando el alumno efectúa estas tareas recurre al análisis de los elementos presentados en ellas, estableciendo relaciones entre los datos, ya que son tareas de mayor exigencia cognitiva, que requieren la atención de los estudiantes en el desarrollo de procedimientos y la comprensión de conceptos e ideas matemáticas; sugieren explícita o implícitamente vías a seguir, que son amplios procedimientos generales donde hay conexiones cercanas a ideas conceptuales subyacentes, en lugar de algoritmos; suelen representarse con múltiples formas y situaciones problemáticas; exigen alto grado de esfuerzo cognitivo y, aunque puede sugerirse un procedimiento, éste no puede realizarse sin reflexión, pues los alumnos necesitan comprender las ideas o conceptos que son la base de la tarea. Cuando los profesores intentan aumentar el nivel de exigencia cognitiva, pueden proponer o sugerir tareas como las siguientes:

"Dividir en cuartos de tal manera que en cada cuarto haya un círculo y un triángulo y dichos cuartos tengan la misma forma"

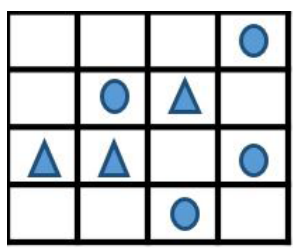

“La figura siguiente representa 3/7, ¿Cómo será el entero?"

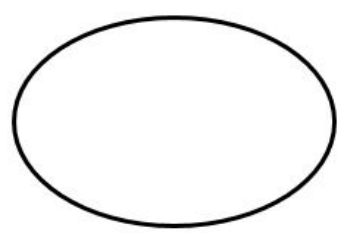

\section{Tareas para hacer matemáticas}

Estas tareas involucran habilidades del pensamiento en las cuales los alumnos visualizan e integran los elementos de una situación problemática propuesta 
que les permite desarrollar un plan que puedan ejecutar; intervienen habilidades como resumir, organizar, diseñar, elaborar, reconstruir, reflexionar, comunicar, empleando de manera flexible sus conocimientos, estrategias y habilidades, y promoviendo una participación autónoma; son tareas de exigencia cognitiva de nivel superior, ya que se exige a los estudiantes que exploren y comprendan la naturaleza de los conceptos o procesos y sus relaciones. Una tarea de este tipo puede ser la siguiente:

"Inventa un problema donde utilices la fracción 4/9 y cuyo resultado sea 1/3"

En estos cuatro tipos de tareas matemáticas, la exigencia cognitiva es distinta y aumenta progresivamente de niveles menos complejos a los de mayor complejidad. Hacemos esta clasificación de tareas bajo el supuesto de que si el profesor, al plantear tareas matemáticas a los alumnos, utiliza la EF a partir de observar, identificar, hacer un diagnóstico, regular su práctica y tomar decisiones, lo que se esperaría es que progresivamente aumente el nivel de exigencia en las tareas que propone en clase; en estos casos, la EF puede ser una herramienta que le permita hacerlo.

La experiencia en esta investigación nos lleva a definir al menos tres funciones de la evaluación formativa que pueden apoyar esta difícil actividad profesional de los docentes: diagnosticar; regular y retroalimentar. Enseguida exponemos estas funciones.

\section{DIAGNOSTICAR, IDENTIFICAR}

De acuerdo con los estándares de evaluación del NCTM, "la evaluación es el proceso de recolección de evidencias acerca del conocimiento del estudiante sobre las matemáticas, su capacidad de uso y su disposición hacia ellas..." (Clark, 2006).

Cuando el profesor plantea una tarea matemática, es conveniente que realice un diagnóstico de lo que saben sus alumnos, lo cual le permite identificar si dicha tarea tiene suficiente potencial para que aprendan el contenido motivo de la clase (García et al., 2011; Martínez-Rizo, 2012). En otras palabras, es necesario que conozca el nivel de conocimiento que sobre el tema tienen sus alumnos, para identificar si la tarea logrará el propósito de hacerlos avanzar en su aprendizaje; un proceso de diagnóstico permitirá obtener información sobre cuál 
tarea habrá de presentarse y cuál será la mejor manera de presentarla. Diagnosticar permite establecer una mejor relación del estudiante con el contenido.

En general, la noción de diagnóstico remite a la acción y efecto de diagnosticar, que de acuerdo con el Diccionario de la Lengua Española (2014) significa "recoger y analizar datos para evaluar problemas de diversa naturaleza"; aquí lo entendemos como la actividad que hace el profesor y que le permite recolectar evidencias para que conozca y reconozca cómo aprenden sus estudiantes, que identifique los procesos o caminos que siguen para aprender un contenido matemático, obteniendo información útil para tomar mejores decisiones en la clase de matemáticas.

Por ejemplo, cuando un maestro interactúa con sus alumnos al resolver una tarea de aritmética y quiere saber qué grado de avance tienen en el proceso de aprendizaje del contenido matemático en cuestión y qué dificultades presentan al trabajar con el mismo, plantea preguntas como: ¿Qué significa el número que escribiste aquí? ¿Cuál es la relación entre estos números? ¿Cómo obtuviste esta cantidad? ¿Qué significa para ti este resultado? Estas preguntas no solo hacen pensar a los niños sobre el contenido en cuestión, sino que dan información importante al profesor sobre los procesos que siguen sus alumnos. A partir de preguntas sobre lo que los estudiantes hacen cuando resuelven una tarea matemática, es posible hacer un diagnóstico de sus procesos de aprendizaje, que eventualmente permitirá al docente tomar decisiones informadas.

\section{REGULAR, INTERPRETAR, TOMAR DECISIONES}

La información es importante para tomar decisiones. Los profesores necesitan obtener información del proceso de aprendizaje que siguen los estudiantes cuando resuelven una tarea matemática, de los posibles errores que pudieran surgir al resolverla, de los posibles caminos que pueden seguir, las preguntas que podrían formular, etcétera. Con esta información el profesor puede tomar decisiones que, eventualmente, le permitirán modificar el nivel de exigencia cognitiva, o adaptar el tipo de las tareas que va proponiendo a sus alumnos. Esto implica, para el docente, un trabajo de monitoreo permanente de lo que hacen los estudiantes cuando resuelven una tarea matemática.

Con base en la información que el maestro obtiene, puede regular la enseñanza; es decir, hacer adaptaciones a su propia práctica. La EF no solamente 
ofrece retroalimentación a los estudiantes para mejorar su desempeño, sino que da retroalimentación a los maestros, con el propósito de que mejoren sus prácticas. Esta función de la EF está centrada en el profesor, que a partir del diagnóstico puede identificar si es necesario repetir el tema usando otras estrategias o si los alumnos ya comprendieron el contenido y decide proponer una tarea más compleja, plantear un problema en lugar de un ejercicio, proponer un proyecto, entre otras cosas.

Cuando un alumno no entendía el significado de un contenido matemático, la justificación tradicional apelaba a las características de los métodos de enseñanza o a las características individuales de los estudiantes diciendo, por ejemplo, que el niño no tenía una buena actitud respecto a las matemáticas, que no estaba motivado, o que simplemente perdió el interés por llegar a una respuesta correcta (Ávila, 2006; Chávez, 2007). "La didáctica de las matemáticas postula que tanto una mala actitud como una falta de motivación -y hasta lo que muchas veces se considera como falta de 'comprensión'- son hechos que se pueden explicar mediante las leyes que rigen el proceso didáctico"(Chevallard, Bosch y Gascón, 1997: 62). Enseñar eficientemente un contenido matemático tiene que ver con el conocimiento de los alumnos y con la didáctica; estos conocimientos y la información que el docente puede obtener de los procesos interactivos cuando monitorea el trabajo de sus estudiantes (diagnóstico) constituyen información valiosa para tomar mejores decisiones, que en la mayoría de los casos son instantáneas.

Con la información que el profesor obtiene de un diagnóstico puede ajustar el contenido matemático para que los estudiantes logren comprenderlo, lo cual no significa bajar la exigencia cognitiva haciendo "más fácil" la tarea. La EF es un proceso que reúne evidencias, se hacen inferencias, se llega a conclusiones y se actúa según ellas; en este sentido es una fuente de sugerencias para el maestro (Clark, 2006: 67), que le permite regular su práctica.

\section{RETROALIMENTAR, INFORMAR, COMUNICAR}

La retroalimentación es el elemento distintivo de la EF (García et al., 2011; Martínez-Rizo, 2012), pues consiste en dar información útil al alumno respecto a su desempeño, valorando lo que puede hacer y apoyándolo a aprender lo que aún no domina (Clark, 2006). La retroalimentación informa sobre el proceso de aprendizaje en tanto el estudiante asume criterios a partir de información que el 
profesor provee. En términos de aprendizaje, hay información útil para el alumno y otra que no lo es. Cuando el profesor dice fijate bien, hay retroalimentación pero no formativa, pues por más que el niño mire con detenimiento el problema, lo que facilita la comprensión no aparece ante sus ojos; es necesaria la intervención del docente con información que ayude al estudiante a superar momentos de confusión.

Cierto tipo de preguntas pueden contribuir a retroalimentar de manera formativa a los estudiantes. Por ejemplo, si un maestro de $5^{\circ}$ grado de primaria pregunta a sus alumnos ¿Cuál número es mayor, el 4.125 o el 4.6? algunos pueden responder que 4.125 y la intervención del profesor puede ser:

No formativa:

- iFíjense bien!

- En los dos números el entero es 4 y es igual

- iSolo tienen que ver la parte decimal!

- iAcuérdense, el primer número después del punto son décimos, es el que tienen que ver!

- Miren el número mayor, es el icuatro punto seis...!
Formativa:

- ¿Por qué creen que el 4.125 es mayor?

- ¿Qué tienen en común ambos números?

- ¿Cómo representarían el .125? ¿Y el .6? [V. gr. dibujando en una cuadrícula]

- ¿Cómo podrías averiguar cuál es mayor?

Para que el maestro pueda ofrecer retroalimentación formativa debe tener claro el propósito de aprendizaje: lo que pretende que sus alumnos aprendan y la manera en que lo harán. En matemáticas, el profesor puede ofrecer retroalimentación: a) Sobre el resultado de un algoritmo, ejercicio o problema; b) Sobre los métodos o estrategias utilizadas para resolver la tarea, los procedimientos o algoritmos empleados; c) Sobre la manera de expresar las ideas que justifican los procedimientos para resolver tareas, es decir, los argumentos.

Los tres puntos anteriores son de suma importancia; sin embargo, los profesores por lo general evalúan el resultado de un ejercicio, problema o algoritmo, y la retroalimentación que dan se reduce a una aprobación o reprobación, que no ofrecen información de calidad a los estudiantes. La retroalimentación centrada en los métodos o estrategias de aprendizaje (b), en los argumentos o ideas que justifican los procedimientos de los alumnos (c), que proporciona un andamiaje o una ayuda para hacer conexiones y continuar en la construcción de conocimiento, son las menos frecuentes, pero podrían ser las que mayor impacto tienen en el aprendizaje.

La EF cumple la función de retroalimentar al proporcionar a los alumnos la oportunidad de mostrar su desempeño con respecto al contenido matemático, 
generalmente a través de preguntas específicas sobre el contenido, pero suficientemente abiertas para proporcionar a la mayoría de los estudiantes la posibilidad de mostrar lo que saben a través de una gama de niveles de sofisticación (Clark, 2006: 72).

\section{METODOLOGÍA}

El trabajo es un estudio exploratorio, sin pretensiones de generalización, cuyo propósito fue caracterizar prácticas de enseñanza de matemáticas a partir de la demanda cognitiva de las tareas propuestas por el docente, e identificar en su intervención, como parte del proceso de enseñanza, elementos de EF que pueden favorecer el aprendizaje de los alumnos.

Diseñamos una guía de observación basada en trabajos previos (Cfr. Ruiz Cuéllar, 2014), ya que la complejidad de lo que ocurre en las aulas hace que, para su estudio, no baste aplicar solo cuestionarios o entrevistas, sino que es necesaria la observación directa, complementada con el análisis de evidencias de lo que sucede en los salones de clase.

Se observaron y videograbaron las clases de dos profesores de primarias públicas que aceptaron participar en el estudio: uno atiende cuarto grado y otro quinto. Ambos son normalistas; el de cuarto es de reciente ingreso al sistema y ha hecho estudios de formación continua, con un diplomado en evaluación y un curso sobre didáctica de las matemáticas; el profesor de quinto grado también continúa su formación y estudia una maestría en la Universidad Pedagógica Nacional. Videograbamos 12 sesiones continuas de clases de matemáticas de cada uno de los docentes para observar la secuencia de enseñanza de un contenido, de principio a fin.

Las sesiones abarcaron tres semanas de clases; para cada sesión el observador llenó La guía de observación de la práctica, donde registramos las actividades del inicio, desarrollo y cierre de la clase, además de datos generales de la escuela y el grupo.

La guía de observación de la práctica permite observar: a) La organización y la estructura de la clase; b) Las acciones para contextualizar el trabajo; c) El tipo de tareas o actividades que los profesores proponen a los alumnos para favorecer el aprendizaje; d) El tipo de interacción alumno-contenido-docente que se promueve a partir de estas tareas; e) El nivel de exigencia cognitiva de las tareas propuestas y f) El tipo de intervención del profesor, enfocándose en 
las prácticas de evaluación (Anexo 1). En la guía se incluyó un apartado para clasificar las tareas y ejemplos de la intervención docente, de acuerdo con el modelo de Smith y Stein (1998). Además de la observación con base en la guía, hicimos entrevista semiestructurada a cada uno de los profesores al finalizar las videograbaciones.

Debido al carácter intensivo del estudio, la información recopilada sobre las prácticas de los dos maestros fue abundante; por razones de espacio solo presentamos algunos episodios que consideramos ilustran lo que se pretende mostrar, en cuanto a la manera en que el maestro puede modificar el nivel de demanda cognitiva de las tareas propuestas en los libros de texto a partir de algunos elementos de la evaluación, con las implicaciones que esto puede tener para el aprendizaje de los alumnos. Aunque, como señalamos, el trabajo no tiene pretensiones de generalización, consideramos que sus hallazgos pueden ser similares a los de muchos otros docentes.

\section{RESULTADOS}

\section{LAS TAREAS DE ALTO POTENCIAL MATEMÁTICO: EL DIAGNÓSTICO, LAS DECISIONES Y LA RETROALIMENTACIÓN AL CENTRO DE LA ACTIVIDAD MATEMÁTICA}

El primer caso de este estudio corresponde a la clase del profesor de cuarto grado, a quien llamamos Juan ${ }^{5}$, la cual se centró en el uso de fracciones para expresar las partes de una colección. Elegimos este episodio porque el maestro propone una tarea de alta exigencia cognitiva y utiliza herramientas de la evaluación formativa, descritas en párrafos anteriores.

El docente presentó una planeación semanal, donde especifica la clase que aquí se analiza:

5 Cambiamos los nombres de los profesores para respetar su anonimato. 
Eje: Sentido numérico y pensamiento algebraico.

Intención didáctica: Que los alumnos identifiquen la cantidad total a partir de la fracción dada.

Lección: 67 de Desafíos matemáticos ¿Cuántos eran?

Contenido: Números y sistemas de numeración.

- Uso de fracciones para expresar partes de una colección. Cálculo del total conociendo una parte.

Adecuaciones: Trabajar con material concreto y con problemas más sencillos para unos equipos.

Propósito: Que el alumno calcule fracciones de cantidades enteras, determine fracciones de una parte dada y el total de una fracción dada identificando los procedimientos aplicables a cada caso y practicarlos para su uso convencional.

\section{Sesión 1 (50 minutos):}

\section{$\mathrm{INIClO}$}

- Decir qué espero que aprendan. Comentar qué aprendieron la semana pasada y escribirlo en una lámina para que lo tengan presente; enfatizar que haremos lo contrario que la semana pasada.

- Organizarlos en grupos de apoyo.

\section{DESARROLLO}

- Escribir dos problemas para identificar qué alumnos se pueden dar cuenta de qué procedimientos utilizar.

- Revisar los problemas utilizados eligiendo el procedimiento correcto para poder resolverlos.

- Comentarlo, y algo que no se haya entendido.

- Realizar el problema del libro de Desafíos matemáticos. Leer qué tienen que hacer y pedir que me lo expliquen.

- Monitorear el trabajo del grupo.

- Revisar los procedimientos usados y marcar en las hojas lo que noté.

\section{CIERRE}

- Revisar y comentar en plenaria lo que noté y cómo resarcir esos errores.

- Escribir en una cartulina el procedimiento que usamos y escribir un título a cada cartulina, anotarlo en sus libretas.

Figura 1. Parte de la planeación semanal del profesor Juan. 
Como puede observarse, desde la planeación el profesor Juan está interesado en que sus alumnos tengan claridad respecto al trabajo que van a desarrollar. En este sentido, Sadler (en Martínez-Rizo, 2012) señala tres elementos de la EF como proceso: el punto de inicio, los saberes que el alumno posee (lo que describe el profesor en el párrafo de Inicio); el de llegada, el referente curricular que se desea dominar (el primer punto del Desarrollo y los dos últimos de Cierre) y el recorrido del primero al segundo, el proceso de enseñanza-evaluación (el segundo y quinto punto de la sección de Desarrollo); desde la planeación del profesor Juan pueden identificarse elementos de la EF.

Aunque en esta sesión el maestro lleva a cabo cuatro actividades, destacamos una tarea matemática (la segunda de cuatro) que clasificamos como de alto nivel de complejidad:

2.1 En el frutero quedan 4/6 de naranjas [que son 7]. ¿Cuántas había en total? ${ }^{6}$

Consideramos esta tarea de alta exigencia cognitiva. Según Smith y Stein (1998) puede categorizarse como tarea de procedimientos con conexión, ya que los alumnos tienen que calcular el total de elementos que integran el referente (total de naranjas en el frutero) a partir de una fracción del mismo.

Esta tarea no permite el cálculo mental o la estimación como estrategias de resolución porque el 7 no es ni múltiplo ni divisor del 6, que aparece como denominador, lo cual obliga a un pensamiento reversible (a partir de la parte, constituir el todo). La fracción 4/6 implicada no es una fracción unitaria, por lo mismo, esta tarea puede ser inaccesible para la mayoría de los alumnos de cuarto grado (SEP, 2011), pues indica que en el frutero sobran 7 naranjas, que corresponden a $4 / 6$.

En este caso el 7 representa dificultades ya que, por ser un número primo, solo puede dividirse entre 1 y 7. A partir de estos elementos la consigna, así planteada, queda fuera de los propósitos de la lección, del contenido curricular y de las herramientas cognitivas de la mayoría de los estudiantes del grado. A pesar de que el profesor diseñó esta tarea, no se percató de esta complejidad hasta que observó lo que hacían sus alumnos en el momento de interactuar en clase.

El profesor hace un rápido diagnóstico de los procesos de resolución de sus alumnos e identifica algunas dificultades cuando utilizan el número 7 para

6 Es la segunda tarea de la clase y es la primera versión de la misma, por lo que se registró como la tarea 2.1 
trabajar en la tarea matemática propuesta, además de que el material que utilizó para representar las naranjas (sopa de pasta) no lo podían partir, por lo cual modifica la tarea comentando al grupo lo siguiente:

Chicos: una naranja está podrida, así que la vamos a tirar a la basura. Ahora tenemos 6 naranjas que representan 4/6 ¿Cuántas naranjas había en total? (2.2)

El maestro (M) decide cambiar el 7 por un 6 para que los estudiantes puedan manipular los números con facilidad y, de ese modo, realizar la tarea, que sigue siendo de alto nivel de complejidad, a pesar de la simplificación hecha por el docente.

M: Lo que ahorita queremos conocer es el entero de una fracción. [Quiere decir que a partir de una fracción debe conocerse el entero] Alumna 1: Hay que sumarlos.

M: Tengo 4/6 que son 6 naranjas ¿̇cómo le van a hacer?

Alumna 1: Si está dividida en 6 partes y tenemos 4 de ellas, nos quedan 2 partes, entonces son 2/3 esos 2/3 los sumamos a 4/6.

M: Ahorita no vamos a hacer equivalencias, pero en lo primero que dijiste estabas bien. Ahora vamos a ver cómo funciona.

M: ¿En cuántas partes está dividido el entero? Lo que queremos saber es cuál es el entero.

Alumno 2: En tercios, van a ser dos de tres.

M: ¿De dónde salieron los tercios? Ahora no vamos a hacer equivalencias, tienes razón pero, ¿en cuántas partes está dividido?

Alumno 3: En seis partes,

M: iMuy bien! En seis partes, ¿̇uántos grupos tengo que hacer?

Alumnos: Seis.

M: ¿Cuántos grupitos tengo en este momento?

Alumno 4: Seis.

M: ¿Seis?

Alumno 5: Siete.

M: ¿Siete? Vean que tengo seis naranjas ¿Cuántos grupos tengo?

Alumno 6: Uno.

M: Recuerden que tenemos dos partes en una fracción, el denominador que son seis partes y cel numerador me dice qué? 
Alumno 1. El denominador es en cuántas partes está dividido el entero y el numerador dice cuántas partes hay del entero.

M: Muy bien ¿de cuántos quedaría cada grupito?

Alumno 2: Ya sé! no quiere que estemos bien, sino quiere que sepamos cómo hacerlo.

M: Sí quiero que sepan cómo hacerlo, pero cuál es la pregunta. Tengo 4 de 6. Hagan 4 grupos.

Alumna 1: Pues entonces tenemos que partir una naranja a la mitad.

M: No los vamos a partir, no vamos a sacar decimales. Tampoco puedo tener seis grupos. Tengo que hacer una equivalencia de esta fracción. ¿Qué equivalencia más pequeña me quedaría de esta fracción?

Cuando el maestro evalúa la situación y observa que sus estudiantes tienen dificultades para avanzar, modifica la estrategia y retoma la decisión que había rechazado: hacer equivalencias, pues lo que esperaba era que sus alumnos hicieran cuatro grupos con las seis naranjas, lo cual implicaba "partir naranjas a la mitad", como sugiere una alumna. Por ello, el docente solicita a los estudiantes que trabajen con tercios para no "partir" las naranjas. Ante esta situación, el profesor argumenta ${ }^{7}$ que "no creía que fuera tan complicado para los niños". En ocasiones los profesores pueden llegar a pensar que los alumnos no encontrarán un procedimiento para resolver el problema, por lo cual hacen simplificaciones, como la que hace el profesor Juan, creyendo que de esta manera están "ayudando" a sus estudiantes, cuando lo que en realidad pasa es que, al disminuir la dificultad, los alumnos no progresan hacia aprendizajes más complejos.

Para "desatorar" la situación, el maestro monitorea el trabajo que realiza el grupo y ofrece una amplia retroalimentación a los alumnos:

M: Vamos a hacer grupos más pequeños. ¿Si cambiamos esta fracción por una más pequeña, cuál sería? [Se refiere a 4/6]

Alumnos: Dos tercios.

M: Cambiamos entonces 4/6 por 2/3 ¿Es igual, no? ¿Es equivalente?

Alumnos: Sí.

M: Ahora, ¿sí puedo dividir esto en tres partes?

Alumnos: Sí.

\footnotetext{
7 En la entrevista semiestructurada posterior a la videograbación de la clase.
} 
M: Pero de esas tres partes, ¿̇uántas partes tenemos?

Alumnos: Dos.

M: Entonces, ¿¿cuántos grupos puedo hacer?

Alumnos: Dos.

M: Hagan dos grupos iguales con sus macarrones. ¿Cuántos grupos tenemos?

Alumno 1: Dos de tres.

M: ¿Cuántos me faltan para tener los tres grupos?

Alumno 2: Tres.

M: No elementos.

Alumno 1: Un grupo.

M: iUn grupo! Ese grupo, ¿̇uántos macarrones tendría?

Alumno 3: Tres.

M: Dibujen los macarrones que les faltan. ¿Ahora sí podemos decir que están completos, son tres y tres [Se refiere al numerador y al denominador]?

Alumnos: Sí.

M: Entonces, ¿̇cuántas naranjas había en total?

Alumnos: Nueve.

M: Entonces, ¿cuál era el entero?

Alumnos: 9.

M: ¿'Seguros?

Alumno 1: Tres novenos.

M: Eso es lo que me faltaría.

El profesor monitorea constantemente el trabajo de los estudiantes y ofrece apoyo para resolver el problema; sin embargo, se centra en la memorización y procedimientos sin conexión, con pocas intervenciones de alta exigencia cognitiva (Tabla 1). Aun sin modificar la tarea por tercera vez y dejar la consigna como "Ahora hay 6 naranjas que representan 4/6 ¿Cuántas naranjas había en total?", probablemente los alumnos habrían resuelto la tarea con preguntas centradas en niveles de complejidad más altos, por ejemplo: ¿Se sabe cuántas naranjas había en el frutero?, ¿cómo podemos averiguar cuántas naranjas había si solo sabemos que hay 4/6?, ¿qué representa en este caso la fracción 4/6? ¿qué nos dice del entero esta fracción?

Como hemos dicho, la EF no ofrece retroalimentación solo al alumno, sino también al maestro, pues permite regular y hacer ajustes en su práctica de enseñanza-evaluación, aunque en este caso el profesor simplifica la tarea para que sus alumnos lleguen a la respuesta que él esperaba. Si bien, con su intervención, 
el profesor no logra llevarlos al nivel más complejo, si hay un avance sutil en la interacción con sus alumnos, que va de intervenciones en el nivel más básico a otros de mayor complejidad (como puede apreciarse en la Tabla 1).

En la primera parte de la Tabla (que es una de las secciones de La guía de observación) se clasifica, con base en el nivel de exigencia cognitiva, la tarea propuesta a los estudiantes, aunque en este caso solo muestra la actividad en cuestión (la $2^{\underline{a}}$ de 4 diferentes actividades que el profesor propuso en la clase de ese día) con las modificaciones que hizo a esta tarea. En la segunda parte se clasifican las preguntas que el docente plantea a los alumnos en los diferentes momentos de la tarea.

Tabla 1. Tipo de exigencia cognitiva de las tareas matemáticas e intervención del profesor Juan.

\begin{tabular}{|c|c|c|c|}
\hline \multicolumn{4}{|c|}{ Nivel de exigencia cognitiva de la tarea matemática: } \\
\hline De memorización & $\begin{array}{l}\text { De procedimientos sin } \\
\text { conexión }\end{array}$ & De procedimientos con conexión & $\begin{array}{l}\text { Hacer mate- } \\
\text { máticas }\end{array}$ \\
\hline & $\begin{array}{l}\text { 2.3 Cambiamos } 4 / 6 \text { por } 2 / 3 \\
\text { ¿Cuántas naranjas repre- } \\
\text { sentan un entero? }\end{array}$ & $\begin{array}{l}\text { 2.1 En el frutero quedan } 4 / 6 \text { de naran- } \\
\text { jas [que son 7] ¿Cuántas había en } \\
\text { total? } \\
2.2 \text { Una naranja se está pudriendo y la } \\
\text { vamos a sacar, entonces ahora tene- } \\
\text { mos } 6 \text { naranjas que son } 4 / 6 \text { ¿Cuántas } \\
\text { naranjas representan un entero? }\end{array}$ & \\
\hline \multicolumn{4}{|l|}{ Intervención docente: } \\
\hline $\begin{array}{l}\text { Enfocada en la memori- } \\
\text { zación }\end{array}$ & $\begin{array}{l}\text { Centrada en procedimien- } \\
\text { tos sin conexión }\end{array}$ & $\begin{array}{l}\text { Centrada en procedimientos con } \\
\text { conexión }\end{array}$ & $\begin{array}{l}\text { Que promue- } \\
\text { ven el hacer } \\
\text { matemáticas }\end{array}$ \\
\hline $\begin{array}{l}\text { 2.1.1 ¿Recuerdan qué } \\
\text { teníamos que hacer para } \\
\text { encontrar el entero? }\end{array}$ & $\begin{array}{l}\text { 2.1.2 ¿Antes de repartir, qué } \\
\text { teníamos que hacer? } \\
\text { 2.1.3 ¿Qué vamos a buscar? } \\
\text { 2.1.4 ¿Ya se fijaron que aquí } \\
\text { no tengo el entero? }\end{array}$ & $\begin{array}{l}\text { 2.1.5 ¿Si las sopas }{ }^{8} \text { son las naranjas, } \\
\text { cuántas naranjas van a tener cada } \\
\text { uno [para hacer sus cálculos]? }\end{array}$ & \\
\hline
\end{tabular}

\footnotetext{
${ }^{8}$ El profesor repartió a cada alumno 6 figuras de sopa de pasta que representaron las naranjas.
} 


\begin{tabular}{|c|c|c|}
\hline $\begin{array}{l}\text { 2.2.2 ¿En cuántas partes } \\
\text { está dividido el entero? } \\
\text { 2.2.4...pero, ¿en cuántas } \\
\text { partes está dividido? } \\
\text { 2.2.7 Recuerden que tene- } \\
\text { mos dos partes en una } \\
\text { fracción, el denominador } \\
\text { que son seis partes y el } \\
\text { numerador ¿que me dice } \\
\text { qué? }\end{array}$ & $\begin{array}{l}\text { 2.2.3 ¿De dónde salieron los } \\
\text { tercios? } \\
\text { 2.2.5 ¿Cuántos grupos tengo } \\
\text { que hacer? } \\
\text { 2.2.6 ¿Cuántos grupitos tengo } \\
\text { en este momento? } \\
\text { 2.2.8 ¿De cuántos quedaría } \\
\text { cada grupito? } \\
\text { 2.2.9 ¿Si cambiamos esta } \\
\text { fracción por una más pe- } \\
\text { queña, cuál sería? }\end{array}$ & $\begin{array}{l}\text { 2.2.1 Tengo } 4 / 6 \text { que son } 6 \text { naranjas, } \\
\text { ¿cómo le van a hacer? }\end{array}$ \\
\hline & $\begin{array}{l}\text { 2.3.1 ¿Si cambiamos esta } \\
\text { fracción por una más pe- } \\
\text { queña, cuál sería? } \\
\text { 2.3.3 Ahora, ¿sí puedo dividir } \\
\text { esto en tres partes? } \\
\text { 2.3.4 Pero de esas tres par- } \\
\text { tes, ¿cuántas partes tene- } \\
\text { mos? } \\
\text { 2.3.5 Entonces, ¿cuántos } \\
\text { grupos puedo hacer? } \\
\text { 2.3.6 Hagan dos grupos } \\
\text { iguales con sus macarrones, } \\
\text { ¿cuántos grupos tenemos? } \\
\text { 2.3.7 ¿Cuántos me faltan } \\
\text { para tener los tres grupos? } \\
\text { 2.3.8 iUn grupo! Ese grupo, } \\
\text { ¿cuántos macarrones tendría? } \\
\text { 2.3.9 Dibujen los macarrones } \\
\text { que les faltan, ċahora sí } \\
\text { podemos decir que están } \\
\text { completos, son tres y tres [Se } \\
\text { refiere al numerador y al } \\
\text { denominador]? } \\
\text { 2.3.10 Entonces, ¿cuántas } \\
\text { naranjas había en total? } \\
\text { 2.3.11 ¿Entonces, cuál era el } \\
\text { entero? }\end{array}$ & $\begin{array}{l}\text { 2.3.2 Cambiamos entonces } 4 / 6 \text { por } \\
\text { 2/3 ¿Es igual, no? ¿Es equivalente? }\end{array}$ \\
\hline
\end{tabular}

Fuente: Elaboración propia, con base en los registros de observación a partir de La guía de observación. (Los números corresponden a los registros; por ejemplo, 2.3 indica que es la actividad 2 de la sesión en su tercera versión o consigna, de acuerdo con los ajustes que hace el profesor). 
La Tabla 1 sintetiza las intervenciones que hizo el profesor Juan, las cuales clasificamos aplicando la taxonomía de Smith y Stein. Puede observarse que las intervenciones del maestro se sitúan mayormente en el nivel de memorización y en el de procedimientos sin conexión, menos en los procedimientos con conexión, y no encontramos alguna que pudiéramos ubicar en el nivel más complejo; sin embargo, como los estudiantes no llegaron rápidamente a la respuesta que el profesor Juan esperaba, simplificó tanto la tarea que finalmente la ubicamos en un bajo nivel de complejidad.

Lo que hace el profesor Juan es un esfuerzo por mantener a los estudiantes en un nivel de razonamiento y reflexión sobre el trabajo matemático, desde que diseña una tarea de alto potencial, pero al pensar que les resulta "difícil" (como lo menciona en la entrevista) simplifica la tarea, disminuyendo la dificultad.

Cuando el maestro realiza un trabajo constante de monitoreo del trabajo de sus estudiantes, recupera información que le permite hacer adaptaciones a su acción; si bien no hay un gran avance hacia niveles más complejos, sin este trabajo de observación, adaptación y retroalimentación difícilmente se podría progresar en el aprendizaje de los alumnos.

\section{Las tareas de baja exigencia cognitiva}

El segundo maestro, que llamaremos Mario, también presenta una planeación para la semana, como sigue: 
Eje: Sentido numérico y pensamiento algebraico.

Intención didáctica: Que los alumnos infieran y describan las características del sistema de numeración maya y las comparen con las del sistema decimal.

Aprendizajes esperados: Análisis de similitudes y diferencias entre el sistema de numeración decimal y el sistema maya.

Lección: 78,79 y 80 del libro de Desafíos matemáticos.

\section{INICIO. ACTIVIDADES PARA INICIAR BIEN EL DÍA}

Plantear problema a resolver individualmente. En una granja avícola se producen 12,384 pollitos, los mismos que serán transportados en cajas con ventilación, en las que caben 96 pollitos. ¿Cuántas cajas se necesitan para transportar a todos los pollitos?

Poner en común las respuestas.

\section{DESARROLLO}

Dividir al grupo en equipos de trabajo.

Retomar la consigna 78 que solamente se inició haciendo reflexión sobre las características de los sistemas de numeración, para qué fueron creados, a partir de lluvia de ideas.

Comentar en equipo las características del sistema de numeración maya a partir de la información que el libro les da.

Revisar la tabla de equivalencias y completarla, con base en las características de sistema, propiciando reflexión sobre por qué decidieron tomar las figuras para representar las cantidades. Poner en común el ejercicio, permitiendo que expresen cómo hicieron para integrar las equivalencias a integrantes de diferentes equipos.

Mostrar lámina con equivalencias si se les dificultó aún comprender y, a partir de esta, propiciar reflexión para completar la tabla o corregirla.

\section{CIERRE}

Escribir con números mayas las cantidades 29, 30 y 31.

Retomar la tabla de la lámina para puntualizar equivalencias.

Figura 2. Parte de la planeación semanal del profesor Mario, correspondiente a la clase aquí descrita. 
Observamos que una parte importante de la planeación de la clase corresponde a las actividades de Inicio, debido a que en las guías para las sesiones mensuales del Consejo Técnico Escolar del ciclo escolar 2014-2015 se sugiere a los profesores "Actividades para iniciar bien el día", sugerencias que, en muchas ocasiones, no tienen que ver con el contenido a trabajar en clase, como ocurre en este caso. Sin embargo, aunque era una actividad rutinaria de inicio, ocupó poco más de la mitad del tiempo de la clase (45 de los 70 minutos que duró la clase). Elegimos este episodio porque, a pesar de que se trata de una tarea rutinaria, queríamos observar si hay elementos de evaluación en este proceso de enseñanza.

De acuerdo con su planeación, el profesor Mario planteó la siguiente tarea:

En una granja avícola se producen 12,384 pollitos, los mismos que serán transportados en cajas con ventilación en las que caben 96 pollitos ¿cuántas cajas se necesitan para transportar a todos los pollitos?

Clasificamos esta tarea como de procedimientos sin conexión, ya que es de tipo algorítmico, para los alumnos de quinto grado que frecuentemente se han expuesto a este tipo de ejercicios que son resueltos con una sola operación (SEP, 2011).

Una vez planteada la consigna, el maestro determinó un tiempo de 15 minutos para que los estudiantes la resolvieran de forma individual, mientras él caminaba entre los lugares de los alumnos observando su trabajo, pero sin dar retroalimentación. Posteriormente se pasó a la puesta en común, en la que ocurrió la siguiente interacción entre maestro y alumnos:

M: ¿Quién quiere pasar a decir cómo lo resolvió? Vamos a ver si es correcto o no es correcto, pero no hay problema. ¿Alguien utilizó suma?

Alumnos: No.

M: ¿Qué utilizaron?

Alumnos: División.

M: ¿Solo división?, ¿Multiplicación? ¿Por ahí vi que alguien había utilizado multiplicación?

Alumno 1: Yo.

M: ¿Alguien pudo haber utilizado dibujos?

9 La Secretaría de Educación Pública (SEP) en México, publica mensualmente unas guías que sugieren actividades de apoyo para la práctica docente. 
Alumnos: No.
M: ¿No pudimos haber utilizado dibujos?

Alumnos: Sí.

Cuando los profesores monitorean el trabajo de los estudiantes, recuperan información que pueden utilizar en el momento en que se desarrolla la actividad matemática para ofrecer retroalimentación inmediata, como el profesor Juan en el ejemplo anterior; o bien con el propósito de obtener información para una puesta en común posterior, como en este caso lo hace el profesor Mario.

M: Vamos a poner atención en cómo lo hicieron sus compañeras. Expliquen cómo lo hicieron. ¿El número de afuera es qué?

Alumno 1: Los pollos.

M: Los pollos que va a haber en cada caja, y el resultado es 129.

Alumno 1: Sí.

M: ¿Alguien más que la haya hecho con división? ¿Quién lo hizo por multiplicación?

Alumno 3: Yo.

M: ¿Cómo le hiciste?

Alumno 3: Multipliqué el 96 por un número que esté cerca del 12,384.

M: ¿Con qué números lo intentaste?

Alumno 3: 13, 15, 16.

M: Te sobró, te faltó, ¿qué pasó?

Alumno 3: Me falta la...

M: Te falta... Les decía que su compañera utilizó otra estrategia ¿Cómo lo hiciste?

Alumna 4: Multipliqué 96 por 12,384, pero me dio un millón y algo.

El diálogo se rompe, perdiendo la continuidad del proceso reflexivo que construía el Alumno 1, quien no logró completar una tarea, pero el profesor suspendió la interacción para atender al Alumno 3. Este resolvía la tarea por aproximaciones sucesivas y el maestro pudo haberle dado retroalimentación formativa con preguntas de alto nivel de exigencia cognitiva, como: ¿Por qué crees que con esos números no te acercaste al 12,348? ¿Qué números crees que te acerquen más? ¿Por qué? Los estudiantes encuentran diversas estrategias de solución y retroalimentarlos con preguntas centradas en niveles más complejos puede posibilitar que establezcan conexiones y logren una mejor comprensión de los contenidos que están involucrados en la tarea matemática. 
M: Como le dio un número muy grande y eso no era posible, decidió hacer una división. Ahora para terminar, ċustedes creen que se pueda hacer multiplicando, como su compañero, por varios números hasta encontrar uno que multiplicado por 96 nos dé 12,384 ?

Alumnos: Sí.

M: ¿Quién está de acuerdo con eso? Levante la mano.

Alumnos: [Levanta la mano la mayoría del grupo.]

M: ¿Quién dice que se puede hacer con una división?

Alumno 5: Pues se puede con las dos ¿no?

M: Sí se puede con las dos. ¿̇Habrá quizá otra?

Alumnos: Sí.

M: Tal vez si a alguien se le ocurre dibujar las cajas de los pollos y dibujar 96 pollos en cada caja... ¿Esto se podría hacer?

Alumnos: Sí.

Alumno 7: Pero es muy tardado.

M: Es muy tardado, ¿entonces que utilizarían?

Alumnos: Multiplicación o división.

Continuando con esta puesta en común que hace el profesor para recuperar las estrategias y procedimientos que los alumnos utilizaron, el maestro se anticipa a las conclusiones que pueden ofrecer los estudiantes, limitando la retroalimentación a algunos consensos.

M: Si tenemos un examen con muchos problemas, elegiríamos lo que nos lleve menos tiempo. Como ya lo hemos visto, hay muchas maneras de resolver un problema, pero ustedes van a elegir el camino que sea más rápido. Tal vez alguien dice: "Lo voy a resolver con una división", pero si no le sale la división puede utilizar una multiplicación, el chiste es llegar al resultado. El resultado, ¿̇uál sería? ¿Cuántas cajas se necesitan?

Alumnos: 129.

M: 129 sería el resultado, no importa si usé multiplicación o división, hasta podría haber usado otra operación. ¿Se les ocurre que pude haber utilizado otra operación?

Alumno 8: La suma.

Alumno 9: La resta.

M: Con suma, ¿cómo sería?

Alumno 8: Sumar 96+96+96+96+96... 
M: ¿Y cuántas veces tendrías que sumar 96?

Alumno 8: 129.

M: Bien, ¿̇y con resta?

Alumno 9: Quitar 96 al número más grande.

M: ¿Y luego?

Alumno 9: Seguir quitando.

M: Seguir quitando hasta llegar al 0 , y ċluego qué voy a hacer? A contar el número de veces que le quité para obtener el resultado.

En este fragmento se plantean algunas preguntas de alta exigencia cognitiva, pero quien responde es el profesor, evitando que el alumno reflexione sobre un aspecto de la división: cómo obtener el cociente a partir de una resta iterada.

Los profesores a veces comentan que a sus alumnos no les gusta pensar, pero intervenciones como estas contribuyen a que los estudiantes no quieran pensar, ya que el maestro es quien piensa y responde por el estudiante.

La siguiente tabla clasifica la tarea matemática que el profesor propuso a sus alumnos, y en la segunda parte, el tipo de intervención del docente:

Tabla 2. Fuente: Elaboración propia, con base en los registros de observación.

\begin{tabular}{l|l|l|l}
\hline Nivel de exigencia cognitiva de la tarea matemática: & \multicolumn{2}{l}{} \\
\hline De memorización & $\begin{array}{l}\text { De procedimientos sin } \\
\text { conexión }\end{array}$ & De procedimientos con conexión & $\begin{array}{l}\text { Hacer mate- } \\
\text { máticas }\end{array}$ \\
\hline & $\begin{array}{l}\text { 1. En una granja avícula } \\
\text { se producen 12,384 po- } \\
\text { llitos, mismos que serán } \\
\text { transportados en cajas } \\
\text { con ventilación con 96 } \\
\text { pollitos ¿Cuántas cajas } \\
\text { se necesitan? }\end{array}$ & & \\
\hline
\end{tabular}




\begin{tabular}{|c|c|c|c|}
\hline $\begin{array}{l}\text { Enfocada en la memori- } \\
\text { zación }\end{array}$ & $\begin{array}{l}\text { Centrada en procedimientos sin } \\
\text { conexión }\end{array}$ & $\begin{array}{l}\text { Centrada en procedimien- } \\
\text { tos con conexión }\end{array}$ & $\begin{array}{l}\text { Que promue- } \\
\text { ven el hacer } \\
\text { matemáticas }\end{array}$ \\
\hline $\begin{array}{l}1.1 \text { ¿Alguien utilizó una } \\
\text { suma? } \\
1.2 \text { ¿Qué utilizaron? } \\
1.3 \text { ¿Solo división?, ¿Multi- } \\
\text { plicación? } \\
1.3 \text { Por ahí vi que alguien } \\
\text { estaba utilizando una } \\
\text { multiplicación. } \\
\text { Entonces, ¿éste es el re- } \\
\text { sultado? } \\
1.6 \text { ¿Alguien más que la } \\
\text { haya hecho con división? } \\
\text { ¿Quién lo hizo por multi- } \\
\text { plicación? }\end{array}$ & $\begin{array}{l}\text { 1.4 ¿Alguien pudo haber utilizado } \\
\text { dibujos? } \\
1.7 \text { ¿Con qué números lo intentaste? } \\
\text { [Multiplicación por aproximación] } \\
\text { 1.8 Te sobró, te faltó ¿qué pasó? } \\
\text { 1.10 ¿Pudieron haber utilizado di- } \\
\text { bujos? } \\
1.11 \text { ¿Este número representa los } \\
\text { pollos? } \\
1.12 \text { ¿Cuántos pollos va a tener } \\
\text { cada caja? } \\
1.13 \text { Es muy tardado [Hacer dibu- } \\
\text { jos] ¿entonces qué harían? } \\
1.14 \text { ¿Cuántas cajas se necesitan? } \\
1.15 \text { ¿Se les ocurre que pude haber } \\
\text { utilizado otra operación? }\end{array}$ & $\begin{array}{l}1.5 \text { ¿El número de afuera } \\
\text { qué es? [Se refiere al divisor] } \\
1.9 \text { ¿Ustedes creen que se } \\
\text { pueda hacer multiplicando, } \\
\text { como su compañero, por } \\
\text { varios números hasta en- } \\
\text { contrar uno que multiplica- } \\
\text { do por } 96 \text { nos dé 12,384? } \\
1.16 \text { ¿Y cuántas veces ten- } \\
\text { drías que sumar } 96 \text { ? } \\
1.17 \text { ¿Y con resta? } \\
1.18 \text { ¿Y luego? [De quitar } \\
96 \text { ] }\end{array}$ & \\
\hline
\end{tabular}

En el caso del profesor Mario, al plantear la consigna de trabajo a sus alumnos, ellos la resuelven de manera individual y aunque el maestro monitorea constantemente lo que hacen los estudiantes, su intervención se centra en animarlos a trabajar con frases como "apúrate", "ponte a trabajar". Con el monitoreo que realiza, el docente obtiene información sobre la cantidad de alumnos que concluyó la tarea y las distintas estrategias y procedimientos utilizados por los estudiantes; una vez que la mayoría terminó, Ileva a cabo la puesta en común para verificar el resultado de la tarea, en este momento sí hay una interacción entre el profesor y los alumnos.

La tarea en cuestión es del tipo de procedimientos sin conexión. En la Tabla 2 apreciamos que las intervenciones del docente se sitúan mayoritariamente en los dos tipos de menor nivel de complejidad, aunque también hay intervenciones de niveles más complejos, lo cual indica que, aunque la tarea no tenga mucho potencial, las herramientas de la evaluación formativa como el diagnostico, retroalimentación y regulación pueden favorecer interacciones en clase centradas en niveles más complejos. 


\section{CONCLUSIONES}

Las prácticas de enseñanza-evaluación en el aula tienen muchos matices, pero dependen fundamentalmente de la intervención del profesor. Una labor profesional de los docentes en la clase de matemáticas es que los estudiantes aprendan los contenidos de manera eficiente, y una manera de hacerlo es elevando el nivel de exigencia cognitiva de las tareas propuestas.

Como presentamos en los resultados, el profesor Juan diseña una tarea de alto nivel de exigencia cognitiva, a partir de la cual hace un importante trabajo de monitoreo que le permite identificar los procesos que van construyendo los alumnos para resolver la tarea matemática.

Haciendo un rápido diagnóstico, les ofrece una abundante y constante retroalimentación; aunque la mayoría de las preguntas que plantea las clasificamos en bajos niveles de exigencia, hay un pequeño progreso hacia niveles más complejos. Que haya una constante y abundante retroalimentación es un elemento fundamental, más no suficiente para la evaluación formativa. Para que esta retroalimentación sea efectiva es necesario que las preguntas que los profesores hacen a los alumnos estén más centradas en los procedimientos y los argumentos que justifican esos procedimientos, y menos en los "resultados" de los problemas o tareas planteadas, además de que es necesario ofrecer al alumno información clara de lo que se espera que haga al encarar una tarea matemática.

Según lo que plantean algunos autores, teóricamente la evaluación formativa contribuye a lograr un progreso en los estudiantes, mejorando su aprendizaje; sin embargo, como observamos en este estudio, cuando los profesores simplifican las tareas matemáticas que proponen a los estudiantes para que lleguen rápidamente a la respuesta correcta, bajan el nivel de exigencia de la demanda cognitiva y piensan que con estas "ayudas" logran que sus alumnos lleguen más rápido a la respuesta correcta, pero lo que sucede en realidad es que les impiden construir la comprensión del contenido matemático, al pensar que los estudiantes, con sus recursos cognitivos, no pueden lograr resolver tareas "difíciles".

Que una tarea matemática implique un desafío para los alumnos, o sea de alta exigencia cognitiva, no es suficiente para asegurar el aprendizaje de un contenido; los contenidos pueden trabajarse de manera profunda o superficial, según el tipo de interacción que el profesor promueva en el aula. Una tarea puede ser de bajo nivel de exigencia, pero el maestro, con su intervención, puede 
hacer preguntas que dirijan a los estudiantes hacia reflexiones de niveles más complejos, y viceversa.

De manera distinta, el profesor Mario planteó una tarea rutinaria, centrada en niveles de baja exigencia cognitiva; sin embargo, a pesar de que sus intervenciones se centran en niveles de baja exigencia cognitiva, hay momentos en que plantea preguntas a sus alumnos centradas en los procedimientos y argumentos; de haber continuado con preguntas más complejas dirigidas a sus estudiantes, podría ofrecerles oportunidades más sólidas para el aprendizaje.

Observar las clases de matemáticas de los profesores a partir de un instrumento que identifique el tipo de tarea matemática y el tipo de interacción que el profesor promueve en el aula, puede permitirles caracterizar su práctica, con el propósito de tomar decisiones respecto al tipo de intervención necesaria en su aula para que sus alumnos aprendan matemáticas.

La EF, como herramienta que permite visualizar el desempeño de alumnos y profesores, sobre todo identificando en dónde están y a dónde quieren llegar, puede beneficiar el trabajo de ambos.

La observación de las prácticas de enseñanza y de evaluación mostró que, aunque las tareas que pueden proponerse a los alumnos con base en libros de texto y otros materiales tengan un alto potencial matemático, la manera real como el maestro conduce la interacción en el aula está muy centrada en desarrollar habilidades de memorización, procedimentales y operatorias. Si bien mantener el trabajo matemático en niveles cognitivos más elevados es una difícil labor para el docente, las herramientas de la evaluación formativa como son el diagnosticar, regular y retroalimentar, pueden facilitarla.

\section{Agradecimientos}

Queremos expresar nuestro agradecimiento a los maestros participantes en este estudio, por abrirnos sus aulas durante largas jornadas y dialogar sobre la enseñanza-evaluación de las matemáticas en la escuela primaria. Agradecemos especialmente a la Dra. Alicia Ávila Storer por la cuidadosa lectura y valiosas sugerencias que ayudaron a mejorar este artículo, y a los árbitros y editores de la revista por el tiempo dedicado a la revisión de este escrito. 


\section{REFERENCIAS}

Ávila, A. (2006). Transformaciones y costumbres en la matemática escolar. Mexico, D. F: Paidós.

Ávila, A. (2004). La reforma realizada: Ia resolución de problemas como vía de aprendizaje en nuestras escuelas. México: Subdirección de Educación Básica y Normal, Dirección General de Investigación Educativa.

Benedicto, J. y Gutiérrez, A. (2015). Análisis de la demanda cognitiva de problemas de patrones geométricos. En Investigación en Educación Matemática XIX. Alicante: SEIEM. (pp. 153-162). España: Sociedad Española de Investigación en Educación Matemática.

Casanova, A. (1998). La evaluación educativa. Mexico, D. F.

Chávez, Y. (2007). Enciclomedia en la clase de matemáticas (Tesis de maestría no publicada). Universidad Pedagógica Nacional. México.

Chevallard, Y., Bosch, M. y Gascón, J. (1997). Estudiar matemáticas: el eslabón perdido entre la enseñanza y el aprendizaje (1ª ed). Barcelona: I.C.E., Universitat de Barcelona: Horsori.

Clark, D. (2006). Evaluación constructiva en Matemáticas: Pasos prácticos para profesores. En Matemáticas. Antología. Secretaría de Educación Pública. México.

García, M., Aguilera, M. A., Pérez, M. G. y Muñoz, G. (2011). Evaluación de los aprendizajes en el aula. Opiniones y prácticas de docentes de primaria en México. México: INEE.

Llinares, S. y Krainer, K. (2006). Mathematics (student) teachers and teacher educators as learners. En Gutierrez, A. y Boero, P. (eds.) Handbook of Research on the Psychology of Mathematics Education: Past, Present and Future (pp. 429-459). Sense Publishers.

Martínez-Rizo, F. (2012). La evaluación en el aula. Promesas y desafíos de la evaluación formativa. México: Universidad Autónoma de Aguascalientes.

National Council of Teachers of Mathematics (ed.). (2014). Principles to actions: ensuring mathematical success for all. Reston, VA: NCTM, National Council of Teachers of Mathematics.

Niss, M. (1993). Assessment in Mathematics Education and Its Effects: An Introduction. En M. Niss (ed.) Investigations into Assessment in Mathematics Education. An ICMI Study (pp. 1-30). The Netherlands: Springer.

Penalva, M. C. y Llinares, S. (2011). Tareas matemáticas en la educación secundaria. En Goñi, J.M. (Coord.) Didáctica de las matemáticas (pp. 27-51) Barcelona, España: GRAO 
Ravela, P., Picaroni, B. y Loureiro, G. (2017). ¿Cómo mejorar la evaluación en el aula? Reflexiones y propuestas de trabajo para docentes. Montevideo, Uruguay: Grupo Magro Editores.

Rizo, F. M. (2012). Investigación empírica sobre el impacto de la evaluación formativa. Revisión de literatura. Revista Electrónica de Investigación Educativa, 14(1), 1-15. Recuperado de http://redie.uabc.mx/redie/article/view/291

Sánchez, M. (2002). A Review of Research Trends in Mathematics Teacher Education. PNA, 5(4), 129-145.

SEP. (2011). Programas de estudio 2011. Guía para el maestro. Educación Básica Primaria. Sexto grado. México: SEP. Recuperado de http://basica.sep.gob.mx/dgdc/sitio/pdf/ inicio/matlinea/2011/sexto_grado.pdf.

Smith, M. S. y Stein, M. K. (1998). Selecting and Creating Mathematical Tasks: From Research To Practice. Mathematics Teaching in the Middle School, 3(5), 344-350.

YOLANDA CHÁVEZ-RUIZ

Domicilio Postal: Porta Canteras 43, Porta Canteras, Aguascalientes, México. C.P. 20200

Teléfono: $\quad 4491754765$ 
ANEXO 1

\section{GUIA DE OBSERVACIÓN DE LA PRÁCTICA}

MATEMÁTICAS

Datos generales:

Escuela:

C.C.T. Grado y grupo:

Número de alumnos inscritos Rango de edades de los alumnos:

Datos de la sesión:

Fecha: Asignatura:

Asistencia de alumnos durante la sesión: Número de sesión observada

Duración de la sesión. Hora de inicio: Hora en que finalizó:

Distribución/acomodo del salón (mobiliario-alumnos) durante la mayor parte del tiempo de la sesión:

Observaciones de eventos o sucesos destacados durante la clase: 


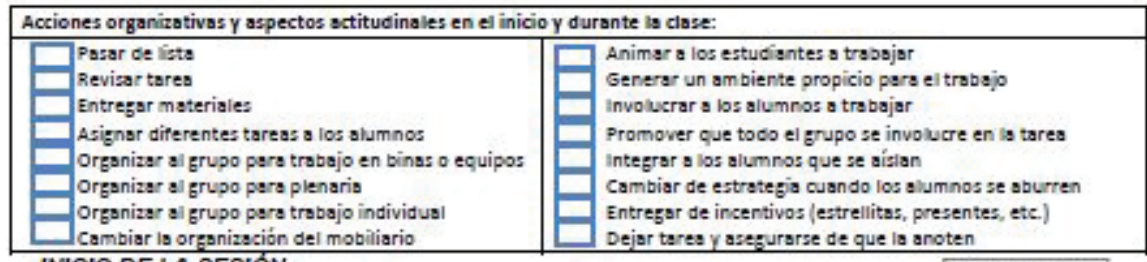

\section{INICIO DE LA SESIÓN:}

Tema o contenido:

Objetivo o aprendizaje esperado:

Criterios de evaluación:

Marcar con una $\checkmark$ la manera en que la o el profesor inicia la clase (pueden ser varias opciones)

\begin{tabular}{|l|l|l|}
\hline 1. & Con la introduccion del tema (sin una ampla explcacion) & \\
\hline 2. & Escriblendo en el pizarron el tema a trabajar & \\
\hline 3. & Enunclando, escriolendo o dictando definiclones & \\
\hline 4. & La explicacion ampla del contenido a trabajar & \\
\hline 5. & Explica lo que tlenen que hacer en la clase sugiriendo procedimientos & \\
\hline 6. & Una consigna o explicacion de io que tienen que hacer & \\
\hline 7. & Una charla mottivadora & \\
\hline 8. & Planteando una sltuacion problematica a resolver & \\
\hline 9. & Otro (especilicar) & \\
\hline
\end{tabular}

Verifica que los alumnos hayan comprendido el trabajo a realizar:

\begin{tabular}{|l|l|l|}
\hline 10. & Preguntando a todo el grupo en general sl hay dudas & \\
\hline 11. & Haclendo preguntas especincas a algunos alumnos sl comprendleron la tarea & \\
\hline 12. & Sollcitando a algunos alumnos que explquen lo que van a reallzar & \\
\hline
\end{tabular}

\section{Descripción del inicio de la clase:}

\begin{tabular}{|c|c|c|c|}
\hline \multicolumn{4}{|c|}{ Tareas matemáticas: } \\
\hline \multicolumn{2}{|c|}{ BAUA DEMANDA COGNITIVA } & \multicolumn{2}{|c|}{ ALTA DEMAANDA COGNITIVA } \\
\hline De memorizoción & $\begin{array}{l}\text { Procedimientos } \sin \\
\text { conexión }\end{array}$ & $\begin{array}{l}\text { De procedimientos con } \\
\text { conexión }\end{array}$ & Hacer matemáticas \\
\hline & & & \\
\hline \multicolumn{4}{|c|}{ Intervención docente: } \\
\hline $\begin{array}{l}\text { Enfocada en la } \\
\text { memorizodión }\end{array}$ & $\begin{array}{l}\text { Centrada en procedimientos } \\
\text { sin conevión }\end{array}$ & \begin{tabular}{|l|}
$\begin{array}{l}\text { Centrada en procectimientos con } \\
\text { conevión }\end{array}$ \\
\end{tabular} & $\begin{array}{l}\text { Que promueven el hacer } \\
\text { motemúticas }\end{array}$ \\
\hline & & & \\
\hline & & & \\
\hline
\end{tabular}




\section{DESARROLLO DE LA SESIÓN}

Marcar con una $\checkmark$ la manera en que la o el profesor desarrolla la clase (pueden ser varias opciones)

Acciones o estrategias para contextualizar el trabajo:

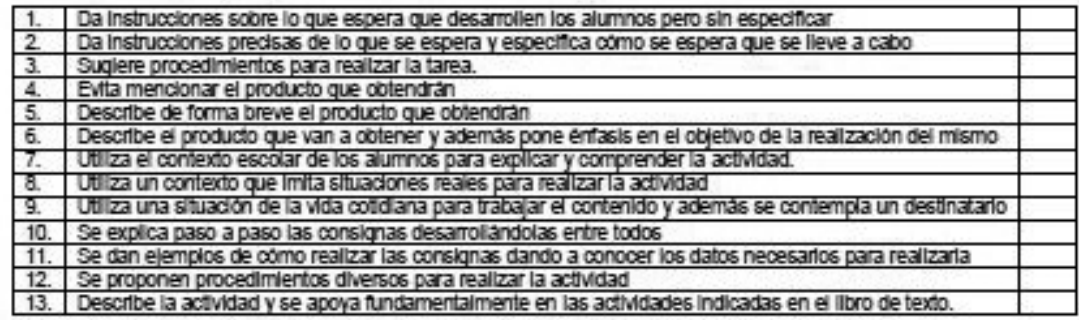

\section{Desarrollo, monitoreo y retroalimentación}

\begin{tabular}{|c|c|}
\hline 13. & $\begin{array}{l}\text { Las actividades desarrolladas consideran condiciones que no pueden ser modifcadas y tenen solo una } \\
\text { solucion lo que no permilte tomar decislones por parte de los aumnos }\end{array}$ \\
\hline 14. & $\begin{array}{l}\text { Las actividades consideran condiciones que no pueden ser modificadas pero permine al alumno blear } \\
\text { aternatvas de solucion y tomar decisiones sobre la solucion al problema respetando las condiciones }\end{array}$ \\
\hline 75. & E prolesor permanece sentado o sale de salon mientras 106 alumnos resueven la activdad. \\
\hline 16. & Se monitorea el trabalo de los estudlantes solo ooservando lo que realizan. \\
\hline 17. & $\begin{array}{l}\text { Se monitorea el trabajo de los estudlantes y los regaŕa o lama la atención para que se fijen y lo hagan } \\
\text { bien. }\end{array}$ \\
\hline 18. & Se monitorea el trabalo de los alumnos corrid endo entores, indicando el procedimiento correcto. \\
\hline 19. & Realiza valoractones soore el trabalo o desempeho de los alumnos como "Elen", "Mal" \\
\hline 20. & E profesor permite que los aumnos se den cuenta de sus entores y les crienta para resolverios. \\
\hline 21. & E profescor da las respuestas de la consigna \\
\hline 22. & Ooserva el trabaio de los alumnos y disminuye la comcielidad de la tarea realizada. \\
\hline 23. & Ooserva el trabaio realizado por los alumnos y aumenta la complelidad de la tarea. \\
\hline 24. & Todos los alumnos se involucran con la tarea. \\
\hline 25. & La mayora de los alumnos se inolucran con la tarea \\
\hline 25. & La mayoria de los aumnos no se involucra con la tarea \\
\hline 27. & E profesor promueve una discusion donde pariapan ios alumnos con argumentos y propuestas. \\
\hline
\end{tabular}

Descripción del desarrollo de la clase:

Focalce ellios metodometodos de evaluacion. Describa las consignas que el profesor va poniendo y focalice en la demanda cognittva. Tome en cuenta la partcipacion de los alumnos.

\begin{tabular}{|c|c|c|c|}
\hline \multicolumn{4}{|c|}{ Tareas matemáticas: } \\
\hline \multicolumn{2}{|c|}{ BAIA DEMANDA COGNIIVA } & \multicolumn{2}{|c|}{ ALTA DEMANDA COGNITIVA } \\
\hline De memorización & $\begin{array}{l}\text { Procedimientos sin } \\
\text { conevión }\end{array}$ & $\begin{array}{l}\text { De procedimientos con } \\
\text { conexión }\end{array}$ & Hacer motemáticas \\
\hline & & & \\
\hline \multicolumn{4}{|c|}{ Intervención docente: } \\
\hline $\begin{array}{l}\text { Enfocodo en la } \\
\text { memorinoción }\end{array}$ & $\begin{array}{l}\text { Centroda en procedimientos } \\
\sin \text { conevión }\end{array}$ & $\begin{array}{l}\text { Centrodá en procedimientos con } \\
\text { conevoón }\end{array}$ & $\begin{array}{l}\text { Que promueven el hacer } \\
\text { motemúticos }\end{array}$ \\
\hline & & & \\
\hline & & & \\
\hline
\end{tabular}


Uso de materiales para la dase (Enliste los materiales que utilizó en clase para la clase):

\section{CIERRE DE LA CLASE}

Marcar con una $\checkmark$ la manera en que la o el profesor desarrolla la clase (pueden ser varias opciones)

\begin{tabular}{|c|c|}
\hline 1. & Organizs una discusión sobre el contenido trabajado, donde participan is mayoría de los slumnos. \\
\hline 2. & Da uns conclusión o síntesis del tems, sin tomar en cuenta el trabajo de los slumnos. \\
\hline 3. & Dicta un resumen del contenido ain considerser las conclusiones o argumentos de los alumnos. \\
\hline 4. & $\begin{array}{l}\text { Extrae información a partir de lo que dicen los alumnos y la organiza para una mejor comprensión, } \\
\text { haciéndola pública en el pizarrón. }\end{array}$ \\
\hline 5. & Pone en común los ejercioios realizados para conocer las respuestas correctas \\
\hline 6. & Pass al lugar de los alumnos a revisar $y / 0$ calificar los ejerócios realizsodos por los alumnos \\
\hline 7. & Les pide que pasen a dejarle al escritorio las sctividades para revisar y/o calificar \\
\hline 8. & Termina la clase por indiscoplins del erupo. \\
\hline 9. & Terminó la clase de manera súbita (por el tiem po u otra actividad programads, sonó la chichsrra, etc.) \\
\hline
\end{tabular}
Observaciones adicionales al cierre:

\begin{tabular}{|c|l|c|}
\hline 10 & $\begin{array}{l}\text { Ante un error, solicits argumentos de los alumnos y retrosliments con preguntss para que revaloren su } \\
\text { idea. }\end{array}$ & \\
\hline 11. & Comete errores motemáticos y no los identifica. & \\
\hline 12. & Comete errores y los corrige snte los alumnos. & \\
\hline 13. & Ante un error, lo corrige y da la explicacón correcta. & \\
\hline 14. & Escucha procedimientos de los alumnos. & \\
\hline 15. & Otra: & \\
& & \\
\hline
\end{tabular}

Describa el cierre de la clase:

\begin{tabular}{|c|c|c|c|}
\hline \multicolumn{4}{|c|}{ Intervención docente } \\
\hline \multicolumn{2}{|c|}{ BAIA DEMANDA COGNITIVA } & \multicolumn{2}{|c|}{ ALTA DEMANDA COGNITIVA } \\
\hline $\begin{array}{l}\text { Enfocondo en la } \\
\text { memorisoción }\end{array}$ & $\begin{array}{l}\text { Centroda en procedimientos } \\
\text { sin conevión }\end{array}$ & $\begin{array}{l}\text { Centrada en procedimientos con } \\
\text { conevión }\end{array}$ & $\begin{array}{l}\text { Que promuleven el hacer } \\
\text { motemúticos }\end{array}$ \\
\hline & & & \\
\hline & & & \\
\hline & & & \\
\hline & & & \\
\hline
\end{tabular}

